\title{
Natural glues and fouling management by interfering with glue curing
}

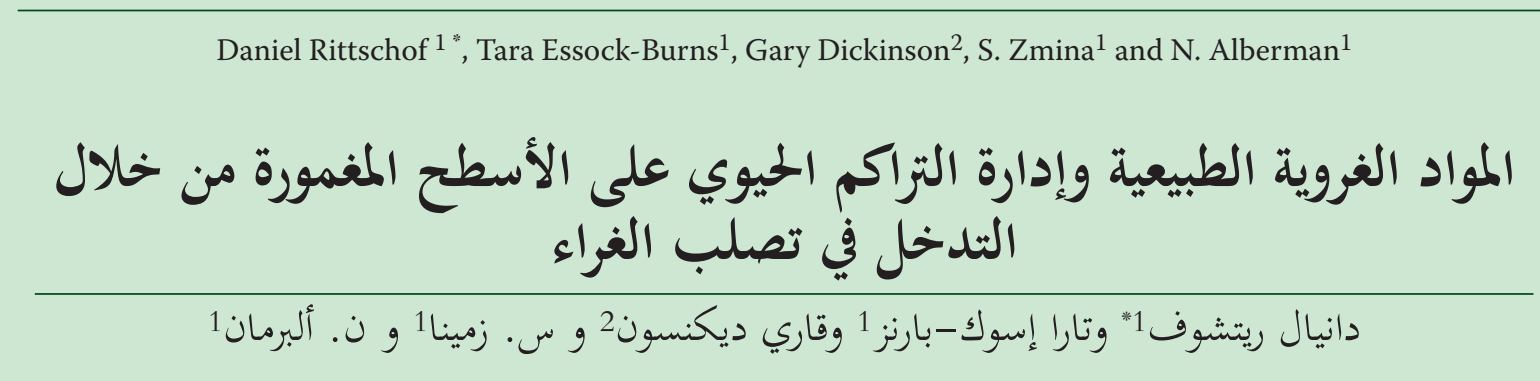

ABSTRACT. Multidisciplinary approaches and modern technology provide insights to glue curing that are stimulating and controversial. Our team applies classic and modern theory and techniques to the study of barnacle glue. Techniques include physical measures, bacteriology, behavior, physiology, biochemistry, microscopy, spectroscopy, tomography, tandem mass spectrometry, molecular biology and proteomics. Theory is grounded in evolution and previous literature. Here, we use data from these techniques to support the hypothesis that barnacle glue curing is similar to blood clotting and propose a model for how glue cures. Similar to blood clotting, barnacle glue curing involves enzymatic activation of precursors and rearrangement of structural molecules to form a crosslinked material. Barnacle larval settlement, bacteriology and biochemical data show glue contains large amounts of small peptides. Their role in glue curing has been overlooked. The peptides comprise 15 to $30 \%$ of partially cured glue. Because they have little secondary structure, the peptides can associate with binding domains on the substrate and interface with the larger, well-described structural proteins known in barnacle glue. Enzymes participate in curing of barnacle glue. Siloxanes impact glue-curing enzymes. They potentiate trypsin activity and inhibit transglutaminase activity. Changing enzyme activity impacts how glue cures. Disrupting the curing process of biological glues is central to effective cleaning strategies for fouling management. Thus silicones that interfere with enzyme activity have potential as additives in easy clean surfaces. The environmental impacts of organosilicones that are generated by biological processes need to be addressed

Keywords: Barnacle; glue; silicone; fouling

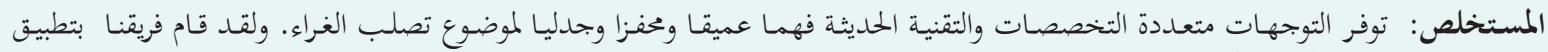

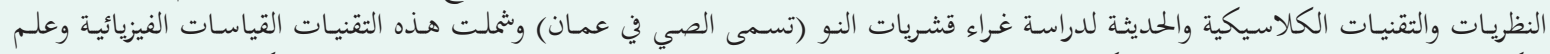

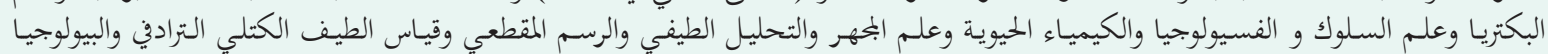

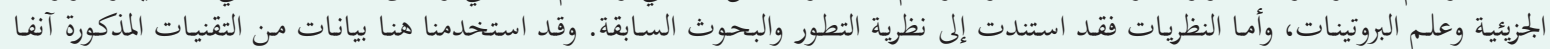

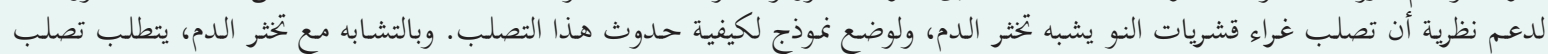

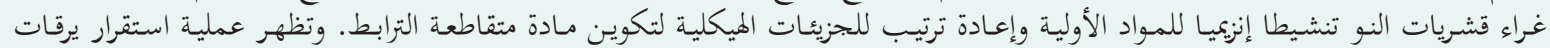

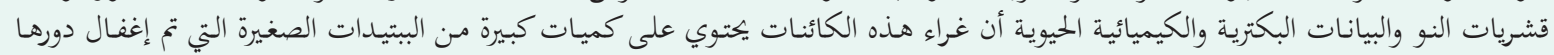

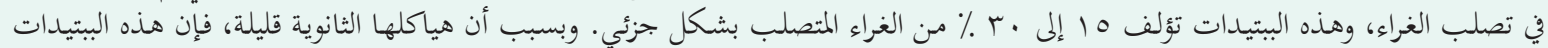

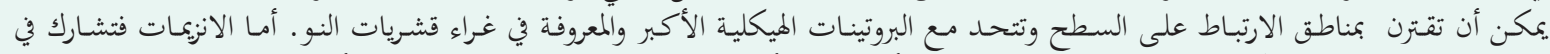

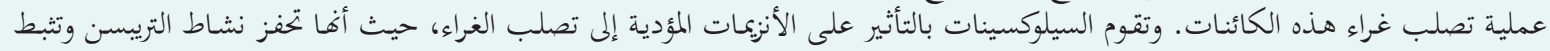

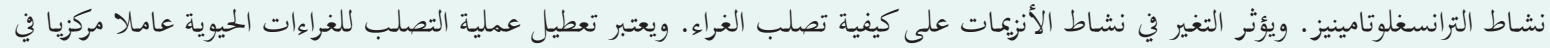

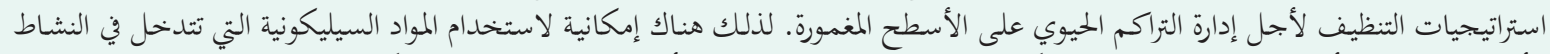

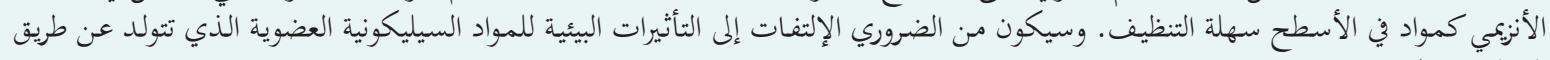
العمليات الحيوية.

$$
\text { الكلمات المفتاحية: قشريات النو (الصي) ، الغراء، السيليكون ، التراكم الحيوي على الأسطح المغمورة. }
$$

\section{Introduction}

$\mathrm{T}$ his paper considers barnacle biology and adhesion; silicone disruption and the potential impacts of silicones as a fouling management tool. We begin with a review of barnacle biology and our

* University Marine Laboratory, Beaufort NC 28516, USA. Daniel Rittschoff $(\triangleright)$ email: ritt@duke.edu

2 Department of Biology, The College of New Jersey، 2000 Pennington Road, Ewing, NJ 08628-0718,USA. work on barnacle glue. There is a much more extensive body of work by many other researchers over the last 50 years that provides the context for our contribution. Here we acknowledge their major contributions by listing the names of at least one member of each group. They include D. Crisp, Graham Walker, P.J. Cheung, G.C. Walker, A. Yule, Larman, Gabbott, K. Kamino, J. R. Saroyan, E. Lindner, C.A. Dooley, E. Holm, D. Wendt, P. Gateholm, G. Swain, E. Singer, A. S. Clare, C. Wang, R. Stewart and our research colleagues, K. Wahl, D, Barlow and D. Burden. It is in the context of this larger body of 
literature that our studies are conducted.

Next, we review what is known of silicones (polydimethyl siloxanes, PDMS) as foul release surfaces and show that barnacle glue cures incompletely on silicones. Using pure enzymes, we support the hypothesis that incomplete glue curing is due to alteration of trypsin and transglutaminase enzyme activities by molecules leaching from silicones.

Polydimethyl siloxane (PDMS) is a central component in most foul-release coatings. PDMS films have a large amount of space within the polymer, which can be loaded with small molecular weight silicone oils (D4 and D5). These low water solubility oils migrate to the surface of the films and can diffuse into natural glues disrupting enzyme activity (Rittschof et al., 2011).

Finally, we discuss studies of the effects of molecules leaching from commercial silicones (iPhone covers and medical grade silicone) on invertebrate and vertebrate embryo development. The impact on embryo development suggests that we should look carefully at potential environmental impacts of silicone leachates.

\section{Barnacle biology and glue curing}

The discussion and simple model that follow need to be reconciled with the larger body of knowledge about barnacle glue and other biological adhesives as well as with the reality of how a barnacle grows. We began the reconciliation by watching barnacles grow with timelapse videos that show growth and morphology in the baseplate. The two short videos are time-lapse videos of the process (supplemental information in Burden et al., 2012).

This first video shows growth of the margin of a rapidly growing juvenile barnacle. The bright areas within the barnacle are regions of active calcification. The very edge is calcification of a lateral plate. The bright spikes about 200 microns from the edge are parts of the calcifying base plate. The edge and base grow continuously when viewed in 30-minute time lapse.

The second video is imaging of growth using timelapse epifluorescence microscopy. Although it cannot be seen in the video, we know that a non-fluorescent "glue" is present under the entire baseplate and at the edge of calcification. As the barnacle grows, detritus

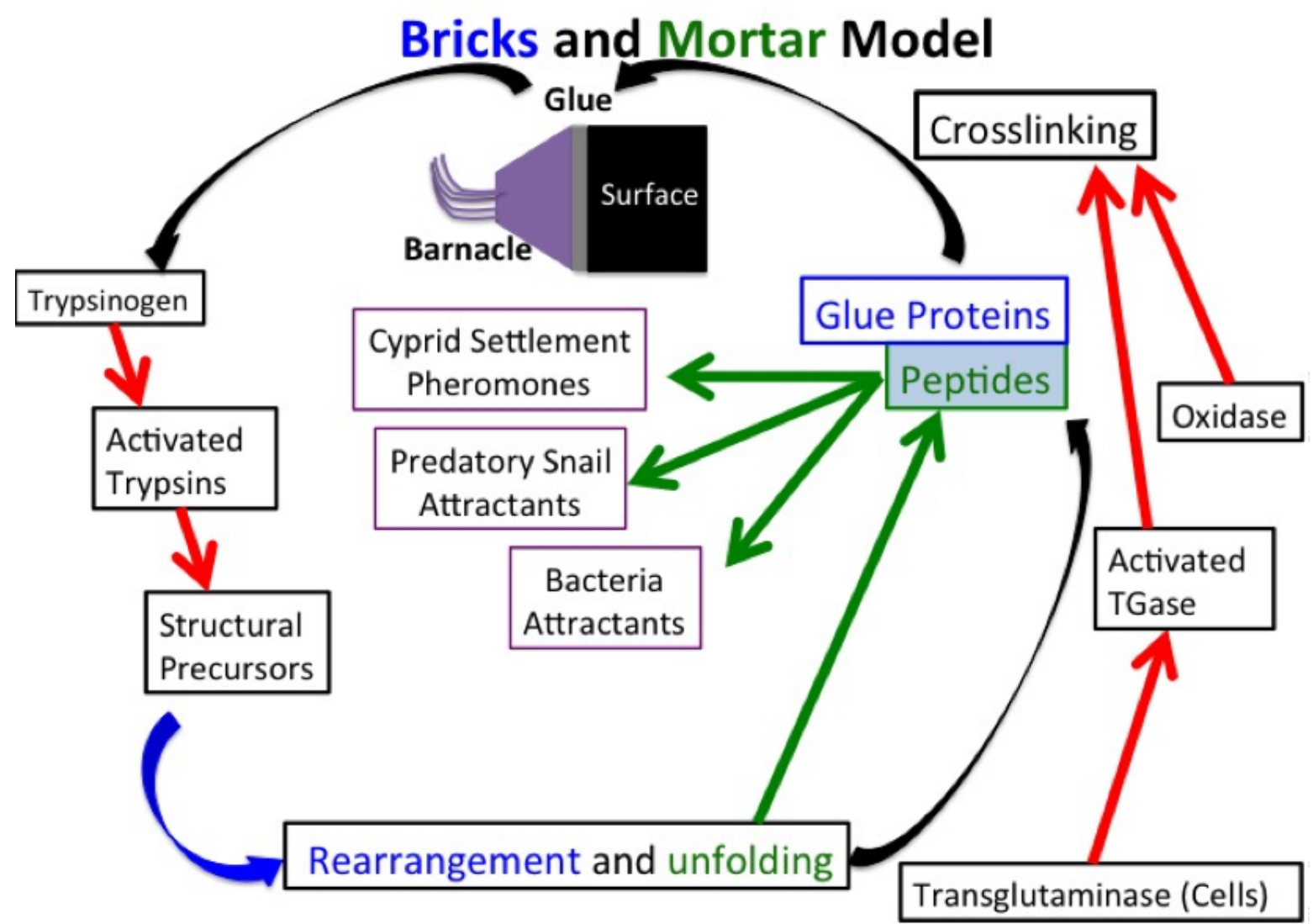

Figure 1. The bricks and mortar model of barnacle glue curing. We call the model bricks and mortar because the present hypothesis is the structural proteins with their secondary and tertiary structure are analogous to bricks, the peptides which have no secondary structure are mortar, the proteolytic are enzymes are activating and the transglutaminase and oxidases cure cement the bricks and mortar and substrate together. Interestingly, peptides that escape crosslinking function as pheromones for barnacle settlement, and predatory snail and bacterial chemoattractants. 
on the surface moves toward the periphery. This video shows that there is a second highly fluorescent episodic deposition through channels that appear intermittently. The next panel shows the less fluorescent glue in the top panel and then the second fluorescent glue in the bottom panel. We hypothesize the second episodic glue deposition is related to the molt cycle, which is consistent with older literature (Crisp and Bourget, 1985).

Finally, the next short video is a tomographic image of the side and base plates of a living barnacle grown on a transparent surface. One can see that there are uncalcified and calcified regions in the base plate and can see the well known concentric growth ring structure. We will test the hypothesis that the second fluorescent solution is important to calcification. As growth slows down and the barnacle continues to molt, the calcification becomes continuous.

We developed hypotheses about barnacle glue curing based on barnacle morphology. From the literature and light microscopy of living barnacles with vital stains we saw that living tissues are found in the channels, calcified base plate and parietal plates (Gohad et al. 2009) X-Ray Tomography showed the channels and living spaces in the plates are very extensive (Wahl et al., 2011). To feed these tissue cells and remove waste the barnacle must be able to circulate the equivalent of a blood. Bourget and others reported that barnacle cuticle often tore as it expanded (Bourget and Crisp, 1975; Crisp and Bourget, 1985). Damage to the cells or plates should cause bleeding. Since barnacles rip their cuticle when they molt and other crustaceans such as lobster, crayfish and crabs, routinely bleed when they molt this seems a reasonable hypothesis. Hardening cuticle following molt is necessary in all crustaceans and leaking of hemolymph is inherent in this process (Terwilliger, 1999). We hypothesized that as blood and hemolymph clot (cure) in aqueous solution that barnacle glue curing could be a form of blood clotting or wound healing as seen in other crustaceans (Söderhäll and Cerenius, 1998; Wang et al., 2001; Theopold et al., 2004; Tassanakajon et al., 2013).

We set out to falsify our hypothesis that barnacle glue curing was a form of wound healing. After 5 years of research, we concluded that the hypothesis was strongly supported by every experiment (Dickinson et al., 2009). Wahl's laboratory at the US Naval Research Lab has subsequently further substantiated and expanded the hypothesis (Barlow et al., 2010; Wahl et al., 2011; Burden et al., 2012, 2014). The new factual expansion is that there are two separate secretions that are barnacle glue. One has no apparent duct structure and is presumably blood delivered through tears in the cuticle when it stretches, as the barnacle grows. The other glue secretion is essentially a spot weld a distance many microns from the glued edge of the expanding plate. The second glue is delivered through capillaries and ducts associated with the molt, which were first reported well over a century ago (Wahl et al., 2011). Barnacles are cyclical animals, growing, molting and reproducing throughout their lives. The timing of these events is critical to understand aspects of adhesion.

Barnacle glue like most other marine glues, but unlike most man made adhesives, forms adhesive bonds and cures in water. Early workers implicated quinone cross-linking as part of glue curing (Lindner and Dooley, 1974). Unlike other well-studied glues, mussel glue (Waite, 1987) and sand castle worm glue (Wang \& Stewart, 2011, 2013), barnacle glue is not rich in L-DOPA but may perform quinone cross-linking through modification of tyrosines (Lindner and Dooley, 1984). Barnacle glue is mainly protein and studies starting with cured glue show the glue is never completely solubilized (Kamino et al., 2006). Kamino and coworkers (2006, 2008) have identified and sequenced a set of structural proteins that are major components of glue. We, and others, are working to understand the assembly of these glue components as they relate to barnacle development and growth.

Barnacle glues are related to wound healing in that coagulation is enzymatically driven. Trypsin-like enzyme activity activates pro-forms of structural proteins, as well as additional serine proteases and crosslinking enzymes (Dickinson et al., 2009). As with trypsin-like enzymes we found with Western blots there is specific binding by antibodies to the crosslinking enzyme, transglutaminase. There is substrate specific transglutaminase activity in curing barnacle glue/blood and specific chemical derivatives followed by acid hydrolysis show about $4 \%$ cross-linking in cured barnacle glue. Hyaline cells (similar to platelets in blood) are hemocytes that appear to rupture and appear to be the source of the transglutaminase. Molecules like heparin that prevent blood clotting and interfere with transglutaminase activity reduce adhesion strength of reattached barnacles. At present we are tracking down antimicrobial, cross-linking, and reactive oxygen species (ROS). In order to understand the transition from glue curing to calcification, collaborators are probing the roles of kinases and phosphoproteins in the glues. We have preliminary data indicating that there are oxidases and peroxidases as well as ROS associated with the growing edge of the barnacle baseplate (Dickinson et al., 2009).

The model below synthesizes all that we know about the role of enzymes, peptides and proteins in glue curing (Fig. 1). This model is based upon our work with glues that contain variable amounts of both barnacle cement secretions, BCS1+ BCS2 (Burden et al., 2012).

\section{Silicones and foul release}

The "Baier adhesive minimum" (Baier, 1970), is a surface energy range where biological glues stick most poorly. At the Baier minimum it is difficult to exclude water, essential for adhesive bond formation (Waite, 2002). At the minimum there is little opportunity for 


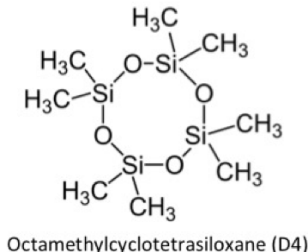

(1)

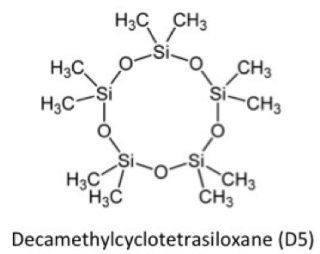

(2) strong ionic bonds or covalent bonds. The surface energy is characterized by mixed, weak Van der waals and weak hydrogen bonding as in the siloxane molecules, D4 and D5 (molecules 1 and 2).

Siloxane films like PDMS (molecule 3) have the surface energy of Baier's adhesive minimum. PDMS was the lining of Barnie Clark's artificial heart and has been studied for decades because of its foul release properties. Interestingly, monolayers of siloxane molecule generated by silane technology have the same critical surface energy, but are not as effective at reducing adhesion of natural glues. It has been hypothesized that the low modulus of PDMS film enables adhesive failure. The hypothesis is supported experimentally (Brady and Singer, 2000). As glue curing is related to blood clot-

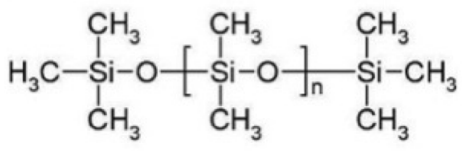

Polydimethylsiloxane (MDnM)

(3)

ting we looked for impacts of PDMS like molecules on blood clotting and found a patent from the 1950s, which claimed that dimethyl silane multilayers interfered with blood clotting in glass tubes.

In the 1990s Swain's group at Florida Institute of Technology did extensive studies on barnacle adhesion to PDMS films (Kavanagh et al., 2005). Studying fracture mechanics of barnacle release from PDMS films they noticed what they described as a "viscous sublayer" (Fig. 2, http://www.youtube.com/FwlHgE3ft-I). In essence, the barnacle was floating on a thin layer of liquid glue. Our group looked at that video, along with the patent on dimethyl silane interruption of blood clotting and hypothesized that molecules leaching out of the surfaces into the biological glues were interfering with curing much as two part epoxy cures incorrectly when mixed in the wrong proportions in our studies of liquid glue. Because we found enzymes, we looked to see if molecules on the surface of silicones would alter enzyme activity.

We found that molecules leach out of silicones (Fig. 3) and that those molecules alter the activity of at least trypsin and transglutaminase enzymes (Rittschof et al., 2011). Interestingly, although the impacts on barnacle glues include differences in morphology and

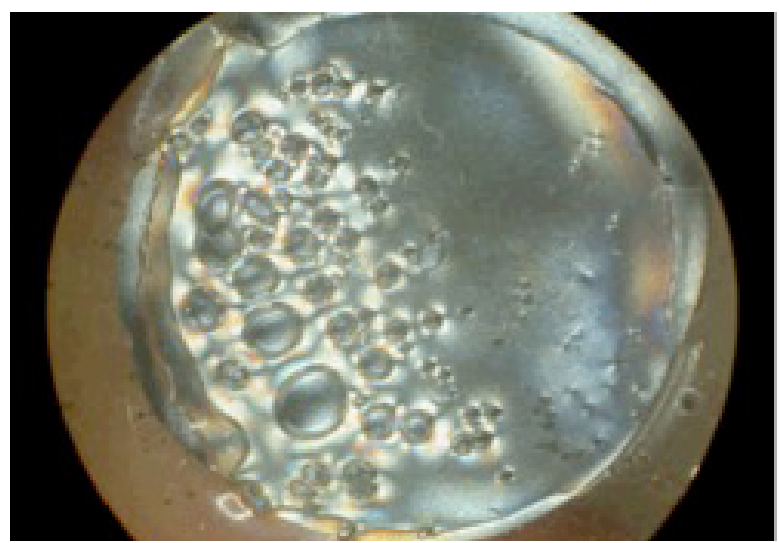

Figure 2. The viscous layer of glue underneath a barnacle attached to silicone (modified from Kavanagh et al., 2005).

adhesion strength between individual barnacles and between different specific silicone films, the general effect (as shown in figure 2), is that molecules leaching out of silicones enhance trypsin like proteolytic activity and inhibit crosslinking by transglutaminase (Rittschof et al., 2011). The result is more small peptides, fewer intact glue proteins and less crosslinking. All effects are consistent with generation of the viscous sublayer observed in the Florida Institute of Technology high-speed video of barnacle detachment (Fig. 2).

This finding is exciting because it provides a mechanism for disrupting the curing of biological adhesives using additives to PDMS and other polymers. Although PDMS and other soft coatings might not be appropriate for a hull or propeller/impeller coating, these coatings could be used where soft coatings would be tolerated such as sensor coatings and coatings that could be cleaned by a water jet rather than a brush. Brushing a soft coating is not a good idea because many attached foulers have calcareous shells, which act as an abrasive and cut into the coating during brushing.

\section{Silicone leachates and organismal impacts}

We discovered during the silicone work that a non-chemical catalyst was conjugating silicone molecules to a steroid (Fig. 3). We hypothesize the catalyst was microbial metabolism of silicone compounds (Rittschof et al., 2011). Knowing that molecules leaching out of silicone alter enzyme activities prompted the question if complex biological systems are impacted by silicone leachates. As embryo development is a very sensitive assay, we chose to test development of an invertebrate, sea urchin embryos, and a vertebrate, Japanese Medaka fish embryos (Feng and Rittschof, 2011). In the first series of experiments we tested foul release coatings and model systems. When that series indicated urchin and fish development were impacted, we moved to more 


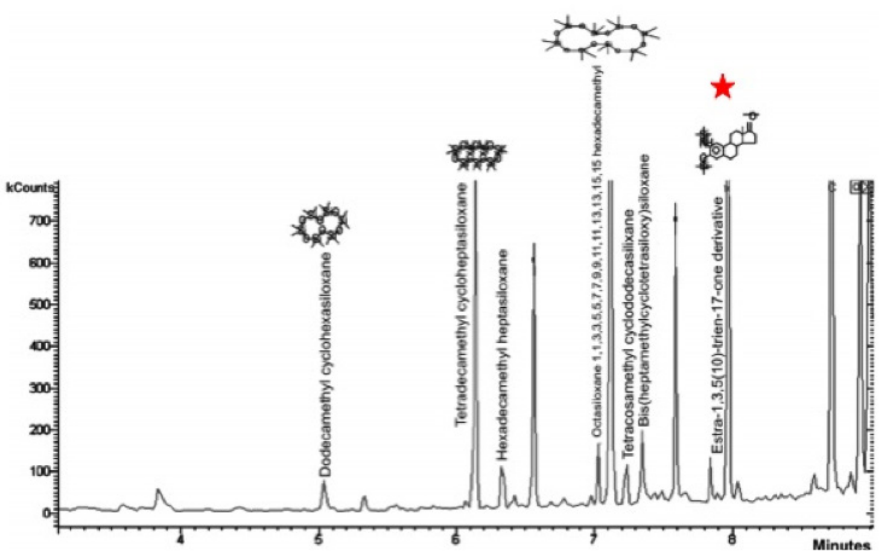

Figure 3. Silicosteroid conjugates detected via GC-MS originating from seawater exposure to commercial T2 silicone. Red star indicates the silicosteroid detected, which was not present in the original coating (Modified from Rittschof et al., 2011).

general silicone surfaces and pure molecules. In that series of experiments we used medaka embryos and tested medical grade silicone, silicone iPhone covers, silicone coffee lids and pure small silicones D4 and D5 (molecules 1 and 2).

For invertebrate and vertebrate toxicology experiments we used a cork borer and cut circles of silicone that fit snugly in the bottom of 24 well polystyrene plates. We added 2 milliliters of NIH embryo rearing medium to each well and then each well received one pre-gastrula developing Medaka egg. Each treatment had 30 replicates. For the small silicone monomers we did a dilution series of D4 silicone $(3.0 \mathrm{mg} / \mathrm{ml}$ and $0.3 \mathrm{mg} / \mathrm{ml})$ in glass scintillation vials. In plate assay and vial assays the embryos were maintained and $25^{\circ} \mathrm{C}$ photographed daily and the water was changed every two days.

We were surprised that all treatments had an effect on Medaka embryo development. Exposing the embryos to silicones reduced mortality presumably due to microbiocidal activity of the leachates in the static conditions. Another consistent impact was the inability of hatched embryos in experimental treatments to inflate their swim bladders. Finally, seen occasionally in the experimental treatments and never in the controls were developmental abnormalities including lack of development of a head, circulatory system disorders, small eyes and only one eye (Fig. 4). The total impact of these treatments is the subject of a manuscript being prepared for submission in a toxicology journal.

All technology has positive and negative impacts. Our ultimate goal is to maximize the positive impacts while minimizing the negative impacts. Often, the negative impacts only become apparent when a particular technology gains market share or societal acceptance. As a consequence, rather than proactive solutions, society is forced into reactive solutions. We suggest that the rational way forward is to begin any development process by including studies that provide insight into potential negative impacts. Adding this knowledge to the research thread that ends in product development would slow the development process down slightly, but result in value added for reducing future human health, environmental health and societal costs.

\section{References}

Baier, R. E. 1970. Surface properties influencing biological adhesion. In R. S. Manley (Ed.), Adhesion in biological systems (pp. 15-48). New York: Academic
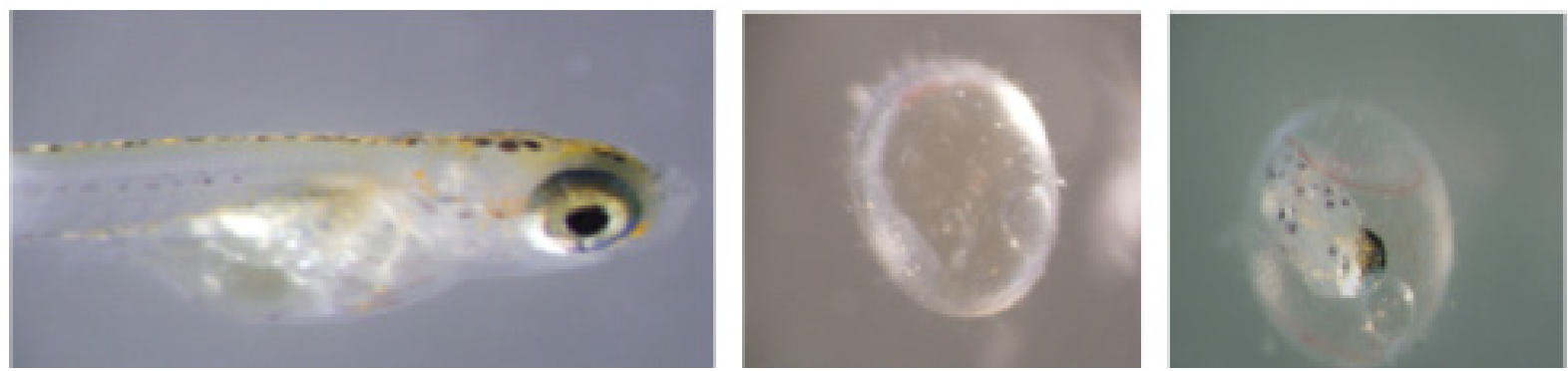

Figure 4. Developmental abnormalities in Japanese Medaka fish embryos exposed to silicones 
Press.

Barlow, D. E., G. H. Dickinson, B. Orihuela, J. L. Kulp, D. Rittschof, and K. J. Wahl. 2010. Characterization of the adhesive plaque of the barnacle Balanus amphitrite: amyloid-like nanofibrils are a major component. Journal of Chemical Ecology, 26(9): 6549-56. doi:10.1021/la9041309.

Bourget, E., and D. J. Crisp. 1975. An Analysis of the Growth Bands and Ridges of Barnacle Shell Plates. Journal of Marine Biological Association United Kingdom, 55(1854): 439-461.

Brady, R. F., and I. L. Singer. 2000. Mechanical factors favoring release from fouling release coatings. Biofouling, 15(1-3), 73-81. doi:10.1080/08927010009386299.

Burden, D. K., D. E. Barlow, C. M. Spillmann, B. Orihuela, D. Rittschof, R. K. Everett, and K. J. Wahl. 2012. Barnacle Balanus amphitrite adheres by a stepwise cementing process. Langmuir, 28(37): 13364-13372.

Burden, D. K., C. M. Spillmann, R. K. Everett, D. E. Barlow, B. Orihuela, J. R. Deschamps, K. P. Fears, D. Rittschoff and K. J. Wahl. 2014. Growth and development of the barnacle Balanus amphitrite: time- and spatially-resolved structure and chemistry of the base plate. Biofouling, 30(7): 799-812.

Crisp, D. J., and E. Bourget. 1985. Growth in Barnacles. In J. H. S. Blaxter \& F. S. Russel (Eds.), Advances in Marine Biology (Vol. 22, pp. 199-244). London: Academic Press Inc.

Dickinson, G. H., I. E. Vega, K. J. Wahl, B. Orihuela, V. Beyley, E. N. Rodriguez, R. Everett, J. Bonaventura, D. Rittschof. 2009. Barnacle cement: a polymerization model based on evolutionary concepts. The Journal of Experimental Biology, 212(Pt 21): 3499-3510. doi:10.1242/jeb.029884.

Gohad N.V., G.H. Dickinson, B. Orihuela, D. Rittschof, and A.S. Mount. 2009. Visualization of putative ion-transporting epithelia in Amphibalanus amphitrite using correlative microscopy: Potential function in osmoregulation and biomineralization. Journal of Experimental Marine Biology and Ecology 380(1-2): 88-98.

Kamino, K. 2006. Barnacle Underwater Attachment. In A. M. Smith \& J. A. Callow (Eds.), Biological Adhesives (pp. 145-166). Berlin, Heidelberg: Springer-Verlag.

Kamino, K. 2008. Underwater adhesive of marine organisms as the vital link between biological science and material science. Marine Biotechnology (New York, N.Y.), 10(2): 111-21. doi:10.1007/s10126-007-9076-3.

Kavanagh, C. J., R. D. Quinn, and G. W. Swain. 2005. Observations of Barnacle Detachment from Silicones using High-Speed Video. Journal of Adhesion: 81(78), 843-868.
Lindner, E., and C. A. Dooley. 1974. Chemical bonding in cirriped adhesive. Proceedings of the Third International Congress on Marine Corrosion and Fouling: 653-673.

Rittschof, D., B. Orihuela, T. Harder, S. Stafslien, B. Chisholm, and G. H. Dickinson. 2011. Compounds from Silicones Alter Enzyme Activity in Curing Barnacle Glue and Model Enzymes. PLoS ONE, 6(2): e16487.

Söderhäll, K., and L. Cerenius. 1998. Role of the prophenoloxidase-activating system in invertebrate immunity. Current Opinion in Immunology, 10(1): 23-28.

Tassanakajon, A., K. Somboonwiwat, P. Supungul, and S. Tang. 2013. Discovery of immune molecules and their crucial functions in shrimp immunity. Fish \& Shellfish Immunology, 34(4): 954-967. doi:10.1016/j. fsi.2012.09.021.

Taylor, J. R. A., and W. M. Kier. 2003. Switching skeletons: hydrostatic support in molting crabs. Science, 301(5630): 209-210. doi:10.1126/science.1085987.

Terwilliger, N. B. 1999. Hemolymph Proteins and Molting in Crustaceans and Insects. Integrative and Comparative Biology, 39(3): 589-599. doi:10.1093/ icb/39.3.589.

Theopold, U., O. Schmidt, K. Söderhäll, and M. S. Dushay. 2004. Coagulation in arthropods: defence, wound closure and healing. Trends in Immunology, 25(6): 289-94. doi:10.1016/j.it.2004.03.004.

Wahl, K. J., R. K. Everett, D. E. Barlow, G. H. Dickinson, and D. Rittschof. 2011. Marine Biofouling: Grasping Barnacle Cement Curing from the Inside Out. 2010 Naval Research Laboratory Review: 89-96.

Waite, J. H. 1987. Nature's underwater adhesive specialist. International Journal of Adhesion and Adhesives, 7(1): 9-14. doi:10.1016/0143-7496(87)90048-0.

Waite, J. H. 2002. Adhesion a la moule. Integrative and Comparative Biology, 42: 1172-1180.

Wang, C. S., and R. J. Stewart. 2011. Localization of the bioadhesive precursors of the sandcastle worm, Phragmatopoma californica (Fewkes). Journal of Experimental Biology, 215(2): 351-361.

Wang, C. S., and R. J. Stewart. 2013. Multipart copolyelectrolyte adhesive of the sandcastle worm, Phragmatopoma californica (Fewkes): catechol oxidase catalyzed curing through peptidyl-DOPA. Biomacromolecules, 14(5): 1607-1617.

Wang, R., S. Y. Lee, L. Cerenius, and K. Söderhäll. 2001. Properties of the prophenoloxidase activating enzyme of the freshwater crayfish, Pacifastacus leniusculus. European Journal of Biochemistry, 268(4): 895902. 